\title{
Preparation of a Paeonol-Containing Temperature-Sensitive In Situ Gel and Its Preliminary Efficacy on Allergic Rhinitis
}

\author{
Kedan Chu ${ }^{1}$, Lidian Chen ${ }^{2}$, Wei Xu ${ }^{1}$, Huang Li ${ }^{1}$, Yuqin Zhang ${ }^{1}$, Weirong Xie ${ }^{1}$ and \\ Jian Zheng ${ }^{1, *}$
}

1 College of Pharmacy, Fujian University of Traditional Chinese Medicine, Fuzhou 350122, China; E-Mails: chukedan@gmail.com (K.C.); xwfjtcm@sina.com (W.X.); lihuang3413@gmail.com (H.L.); zyqfj@hotmail.com (Y.Z.); xweirong3413@gmail.com (W.X.)

2 Faculty of Rehabilitation Medicine, Fujian University of Traditional Chinese Medicine, Fuzhou 350122, China; E-Mail: clidianlab@gmail.com

* Author to whom correspondence should be addressed; E-Mail: zjfjtcm@gmail.com; Tel./Fax: +86-591-2286-1661.

Received: 10 October 2012; in revised form: 25 February 2013 / Accepted: 26 February 2013 / Published: 22 March 2013

\begin{abstract}
In this paper, the optimal composition of a paeonol temperature-sensitive in situ gel composed of poloxamer 407 (P407) was determined, and a preliminary study of its effect on allergic rhinitis was performed. The optimal composition of the paeonol temperature-sensitive in situ gel included 2\% paeonol inclusion, 22\% $\mathrm{P} 407,2 \%$ poloxamer 188 (P188) and 2\% PEG6000, as assessed by thermodynamic and rheological studies. The toad palate model was employed to study the toxicity of the paeonol temperature-sensitive in situ gel on the nasal mucosa. The result of this experiment showed low toxicity to cilia, which allows the gel to be used for nasal administration. The Franz diffusion cell method was used to study the in vitro release of paeonol and suggested that the in vitro release was in line with the Higuchi equation. This result suggests that the paeonol could be absorbed into the body through mucous membranes and had some characteristics of a sustained effect. Finally, the guinea pig model of ovalbumin sensitized allergic rhinitis was used to evaluate the preliminary efficacy of the gel, with the paeonol temperature-sensitive in situ gel showing a significant effect on the guinea pig model of sensitized allergic rhinitis (AR).
\end{abstract}

Keywords: inclusion complex of paeonol and $\beta$-cyclodextrin; in situ gel; allergic rhinitis; rheological studies; thermodynamic study; nasal mucosal toxicity 
Abbreviations: P407, poloxamer 407; P188, poloxamer 188; AR, allergic rhinitis; TCM, Traditional Chinese Medicine; $\beta$-CD, $\beta$-cyclodextrin; DSC, differential scanning calorimetry; LTE4, leukotriene E4; IgE, immunoglobulin E.

\section{Introduction}

Allergic rhinitis (AR), also known as hyperaesthetic rhinitis, is an allergic inflammatory disease that is caused by a variety of allergens. AR is an allergic reaction that often occurs in nasal passages during the spring, autumn and winter [1]. The clinical symptoms of AR are different in different people, but the main symptoms are nasal itching, sneezing and a runny, blocked nose. The intranasal administration of drugs has long been used for the treatment of rhinitis and nasal congestion. Intranasal administration can overcome the side effects that occur in the gastrointestinal tract and the hepatic first-pass effect. Moreover, drugs are absorbed better, because of the abundant blood and lymphatic capillaries under the nasal mucosa. Because of these properties, intranasal administration can effectively improve the bioavailability of drugs. Intranasal administration has been reported to reach similar blood concentrations as intravenous administration [2]. Most of the commercially available nasal preparations are now sprays. The scavenging effect of nasal cilia leads to a very short drug residence time on the human nasal mucosal surface (only 15-30 min) [3], which affects the clinical efficacy to some extent. Therefore, a high research value and various application prospects surround the development of clinically efficacious and safe nasal preparations.

An in situ gel is made of polymer materials that have a solution or semisolid state that responds to external stimuli at the administration site. These gels also have conformations that can undergo reversible conversion to form a semisolid or solid preparation [4]. In situ gel types include thermo-sensitive, ion-activated and electric-sensitive, magnetic field-sensitive, ultrasonic-sensitive and chemical material-sensitive varieties. The most extensive and mature type of in situ gel currently used in research is the gel that reacts to changes in temperature, called a "temperature-sensitive in situ gel". A temperature-sensitive in situ gel is liquid or semisolid at room temperature and congeals into gel as the temperature increases from room temperature to body temperature, which results in good adhesion and slow release effects $[5,6]$. The gel has a three-dimensional network structure that is hydrophilic and has the advantages of good biocompatibility, simple preparation, easy use, good mucosal tissue affinity and long residence time. Poloxamer 407 (P407) is one of the most widely used and most thoroughly studied polymer materials in the temperature-sensitive in situ gel family [7]. The structure of P407 is shown in Figure 1b; it is a non-ionic triblock copolymer (PEO-PPO-PEO) that consists of approximately $70 \%$ polyethylene oxide (PEO) and 30\% polypropylene oxide (PPO), and it has an average molecular weight of approximately 11,500. P407 is soluble in water and has surface activity and a good safety level. Moreover, a certain concentration of P407 has a reverse thermal gelling property that causes it to be a liquid at low temperature and a gel when the temperature rises. Therefore, temperature-sensitive in situ gels that use P407 as a main component were used in this paper.

In 2001, the World Health Organization recommended glucocorticoids as the preferred drug for treatment for AR. Although the safety of nasal corticosteroid treatment has been widely recognized by the industry, its systemic side effects are still the main obstacle to its wider applications in the clinic. It has been reported that Traditional Chinese Medicine (TCM) has great advantages in adjusting immune 
function, and people are now giving TCM more and more attention for its positive and long-lasting effects on AR and its limited number of side effects.

Paeonol (as shown in Figure 1a) is the main active component of the root bark of Paeonia suffruticosa Ardr. and Paeonia lactiflora Pall. and the root of the entire Cynanchum paniculatum (Bunge) Kitag. plant. Paeonol is a low molecular weight phenolic compound that is bitter and acrid in taste. It is volatile, has poor solubility in water and has various analgesic, antioxidant and anti-inflammatory pharmacological effects [8]. The main paeonol preparations currently for sale are paeonol injections, paeonol tablets and paeonol ointments. These formulations are used for the clinical treatment of eczema, cardiovascular diseases [9], allergic rhinitis [10,11], gynecological diseases and other ailments [12].

Figure 1. The chemical structure. (a) paeonol; (b) the triblock copolymer (polyethylene oxide (PEO)-polypropylene oxide (PPO)-PEO) block copolymer.

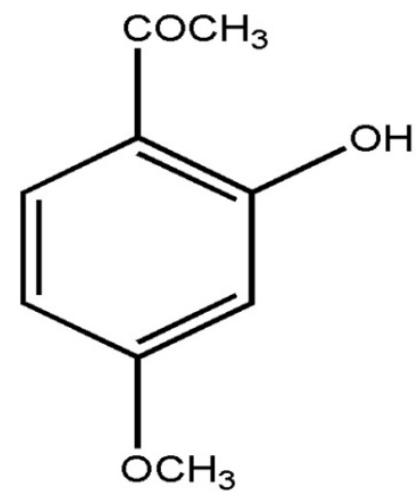

(a)

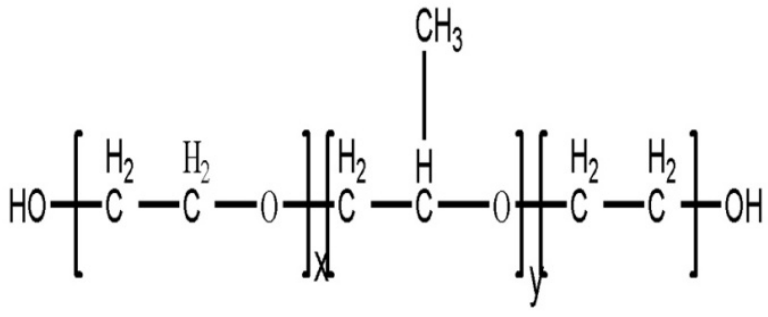

(b)

In this study, paeonol was included by $\beta$-cyclodextrin $(\beta-C D)$, and an optimum formulation of a paeonol temperature-sensitive in situ gel that was primarily composed of poloxamer 407 was sieved out through thermodynamic and rheological studies at the same time. We not only studied the in vitro release and nasal ciliary toxicity of the gel, but also examined the therapeutic effect that the nasal delivery of the paeonol- $\beta-C D$ temperature-sensitive in situ gel has on AR. The objective of this paper was to provide a new TCM preparation to effectively treat AR.

\section{Results and Discussion}

\subsection{The Inclusion Complex of Paeonol and $\beta-C D$}

Because paeonol is volatile and has poor water solubility, the formation of complexes with $\beta-C D$ protects the paeonol by cutting off contact with the surrounding solvent, which could effectively prevent oxidation and volatilizing, thus increasing its stability. Two terminals of paeonol and $\beta$-CD inclusion complex were water soluble, and the capsule interior was lyophobic-properties that could increase the water solubility, the stability and bioavailability.

The infrared spectrograms of paeonol, $\beta-\mathrm{CD}$, the physico-compounds of paeonol and $\beta-\mathrm{CD}$ and the inclusion complex of paeonol and $\beta-C D$ are shown in Figure 2. Figure 2a shows that paeonol had an absorption in the range of $1504.6-1627.6 \mathrm{~cm}^{-1}$ and $1911.9-2099.4 \mathrm{~cm}^{-1}$, due to the carbonyl conjugate 
system in the molecular structure of paeonol. $\beta$-CD (Figure $2 b$ ) had strong absorption at $3200-3500 \mathrm{~cm}^{-1}$ and $800-1200 \mathrm{~cm}^{-1}$. The basic diffraction peaks of the physico-compounds of paeonol and $\beta-\mathrm{CD}$ (Figure 2c) had some features in common with the diffraction peaks of paeonol and $\beta-C D$. The physico-compound absorbed in the range of $3300-3400 \mathrm{~cm}^{-1}, 1504.6-1627.6 \mathrm{~cm}^{-1}$ and $800-1200 \mathrm{~cm}^{-1}$ and had melting point peaks for both paeonol and $\beta-\mathrm{CD}$. This indicated that the paeonol and $\beta-\mathrm{CD}$ were simply mixed and not interconnected. However, the inclusion complex of paeonol and $\beta-C D$ (Figure 2d) was different. Some of the diffraction peaks of the inclusion complex of paeonol and $\beta$-CD disappeared, due to the screening effect that paeonol has on $\beta-C D$. However, there was still a peak characteristic of paeonol at $1627.6 \mathrm{~cm}^{-1}$. This may be because there was a small amount of residual paeonol in the solution, as the above results fully prove that paeonol was in the cavity of $\beta-\mathrm{CD}$ and the inclusion complex had already constituted a new object.

Figure 2. The infrared spectrograms of (a) paeonol, (b) $\beta$-cyclodextrin $(\beta-\mathrm{CD})$; (c) the physico-compounds of paeonol and $\beta-\mathrm{CD} ;(\mathbf{d})$ inclusion complex of paeonol and $\beta-\mathrm{CD}$.
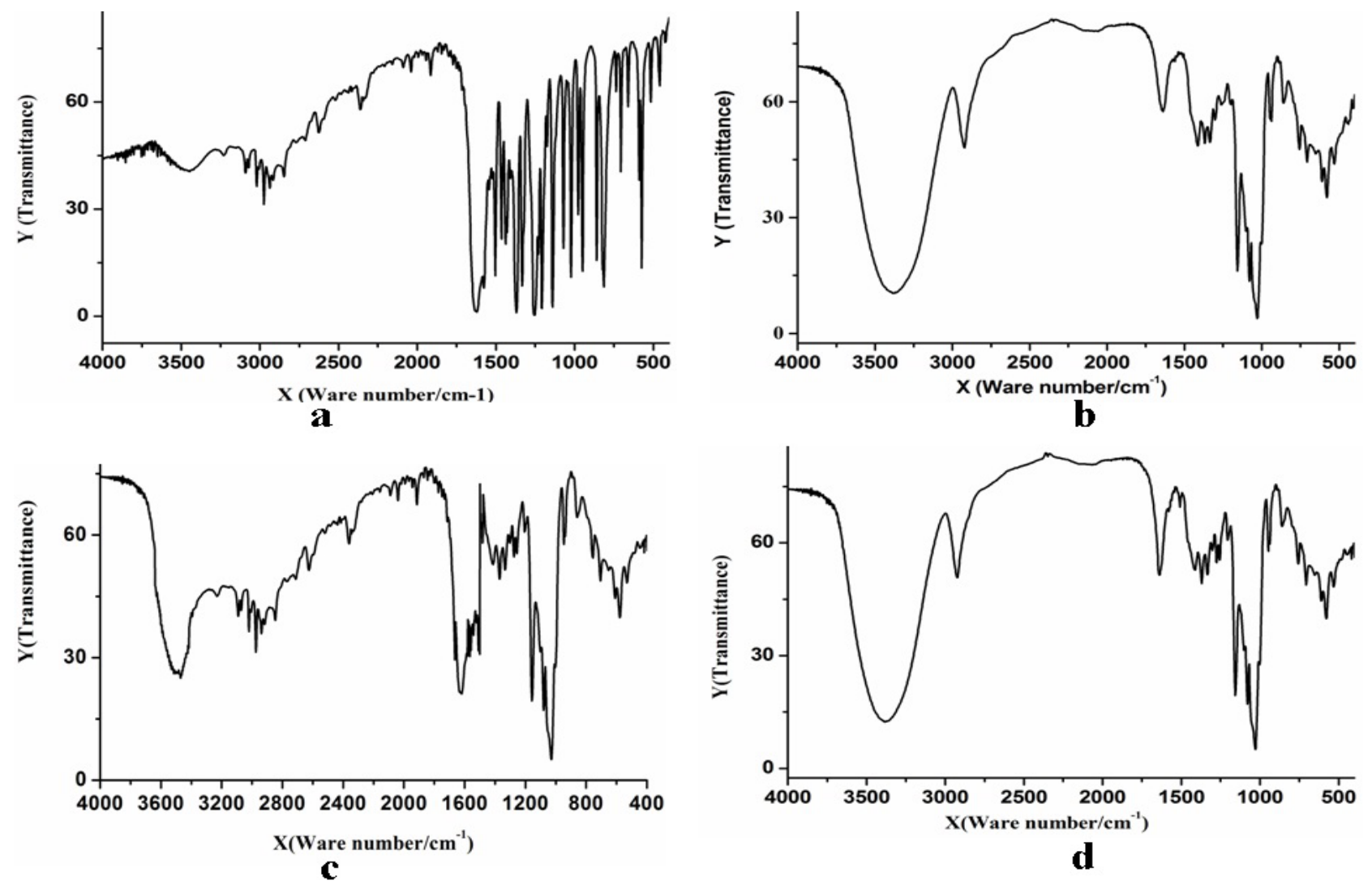

Figure 3 shows the differential thermal curves of paeonol (a), the physico-compounds of paeonol and $\beta-C D(b), \beta-C D(c)$ and the inclusion complex of paeonol and $\beta-C D(d)$. Paeonol had strong absorption peaks approximately $50{ }^{\circ} \mathrm{C}$, which corresponded to its melting point peaks. The melting point peaks of $\beta$-CD appear at approximately $100{ }^{\circ} \mathrm{C}$ and $300{ }^{\circ} \mathrm{C}$, and the physico-compounds of paeonol and $\beta-\mathrm{CD}$ had peaks characteristic of both paeonol and $\beta-\mathrm{CD}$, possibly indicating that the two were only mixed and not interconnected. The differential thermal curves for the inclusion complex of paeonol and $\beta-\mathrm{CD}$ showed that the strong absorption peaks at approximately $50{ }^{\circ} \mathrm{C}$ had disappeared and that there was a new peak at approximately $90{ }^{\circ} \mathrm{C}$. It could be inferred that the inclusion complex 
of paeonol and $\beta-\mathrm{CD}$ was different from the physico-compounds of paeonol and $\beta-\mathrm{CD}$, because an inclusion complex of paeonol and $\beta-\mathrm{CD}$ had formed.

Figure 3. The differential thermal curves of the different samples. (a) paeonol; (b) the physico-compounds of paeonol and $\beta-\mathrm{CD}$; (c) $\beta-\mathrm{CD}$; and (d) the inclusion complex of paeonol and $\beta-C D$.

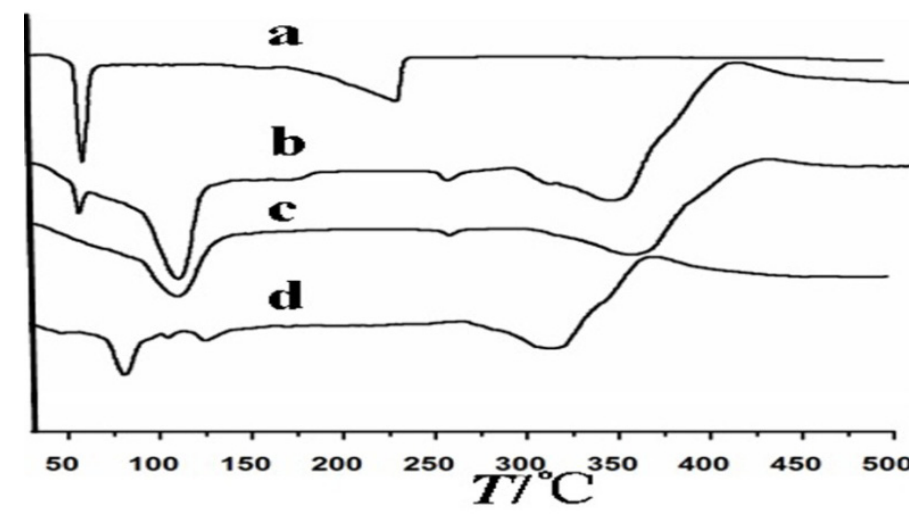

\subsection{Composition Screening of the Paeonol Temperature-Sensitive In Situ Gel}

P407 is a heat-shrink non-ionic surfactant, and its gelling mechanism is considered to be caused by tangles and stacks among the micelles when the temperature increases. A pure P407 solution can generally form a gel at temperatures below $30{ }^{\circ} \mathrm{C}$. This is not the ideal gelling temperature, but the appropriate gelling temperature can be obtained by adjusting the ratio of PPO block and PEO block. Combining P407 with other auxiliary materials, such as poloxamer homologues (like poloxamer 188) and polyethylene glycol, can improve gel properties. For example, polyethylene glycol can increase the gelling temperature and reduce the amount of poloxamer required [13]. Therefore, P407 served as the substrate and P188 and PEG6000 served as temperature regulators for the preparation of the paeonol temperature-sensitive in situ gel. $T_{1}, T_{2}$ and $\eta$ were chosen as indices for screening the composition.

\subsubsection{Influence of $\mathrm{P} 407$ on $T_{1}, T_{2}$ and $\eta$}

The gelling temperature of $\mathrm{P} 407$ had a strong concentration dependence and a high concentration of P407 (18\%-25\%) was usually used to ensure sensitivity to temperature changes. The proper amounts of paeonol inclusion, 4\% P188 and 2\% PEG6000 were put into the solution and P407 was added to prepare a $16 \%, 18 \%, 20 \%, 22 \%$ or $24 \%$ solution $(w / w)$. The results for $T_{1}, T_{2}$ and $\eta$ are shown in Figure $4 a, b$. 
Figure 4. The effects of the concentration of poloxamer 407 (P407), poloxamer 188 (P188) and PEG6000 on $T_{1}, T_{2}$ and $\eta$. (a) the effects of different concentrations of P407 on $T_{1}$ and $T_{2}$; (b) the effects of different concentrations of P407 on $\eta$; (c) the effects of different concentrations of P188 on $T_{1}$ and $T_{2} ;$; (d) the effects of different concentrations of P188 on of $\eta$; (e) the effects of the different concentrations of PEG6000 on $T_{1}$ and $T_{2}$; (f) the effects of the different concentrations of PEG6000 on $\eta$.

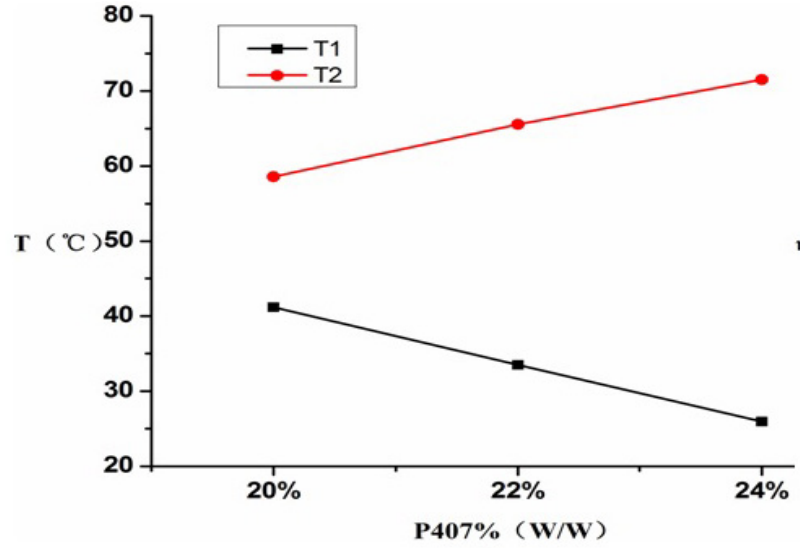

a
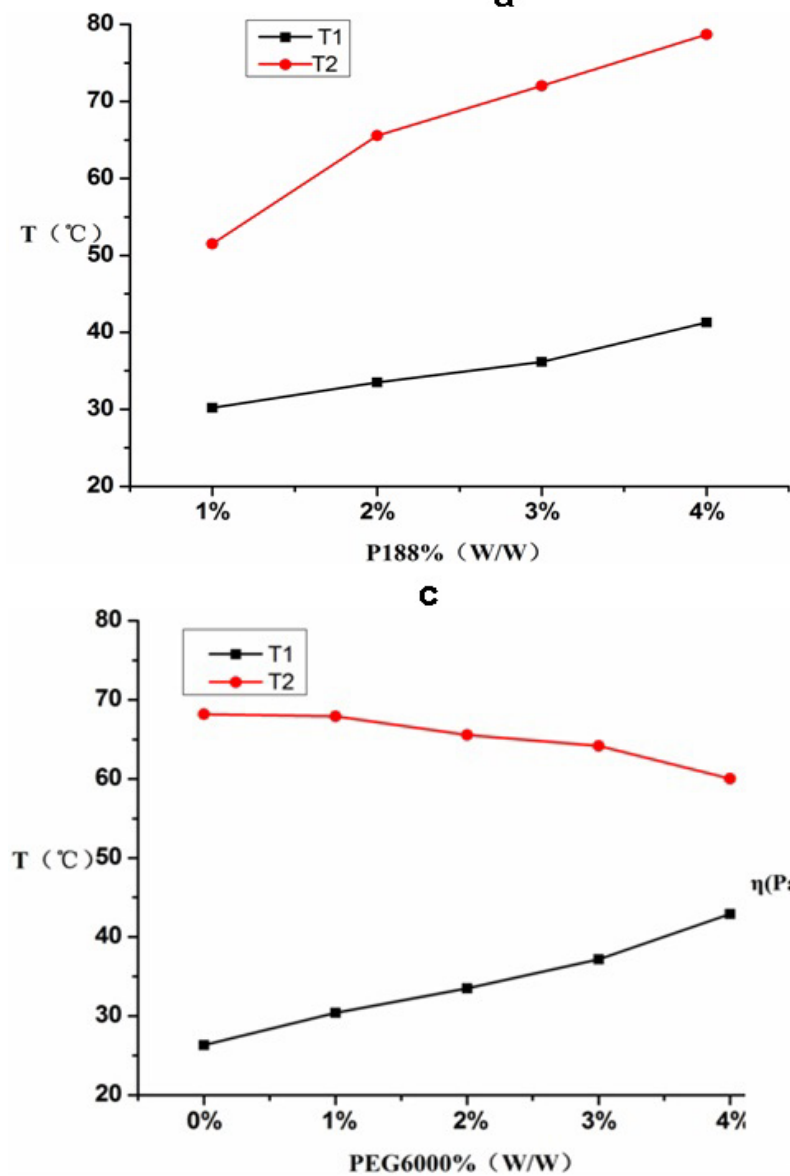

e

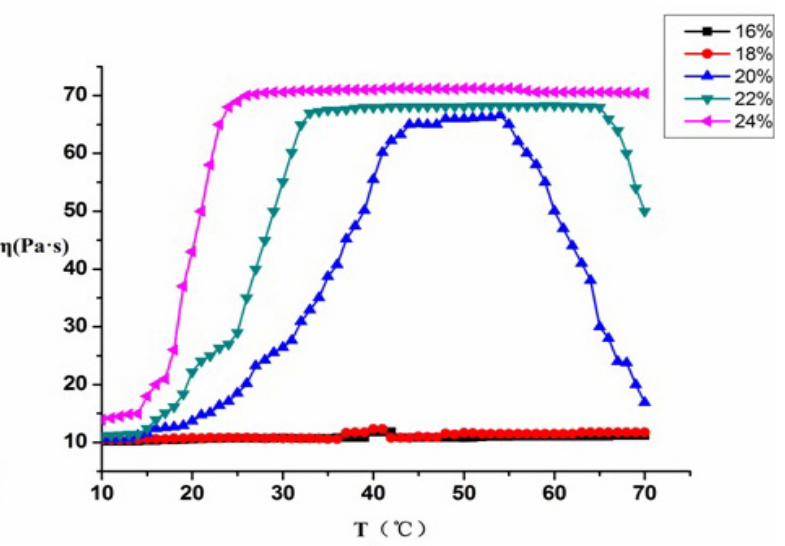

b

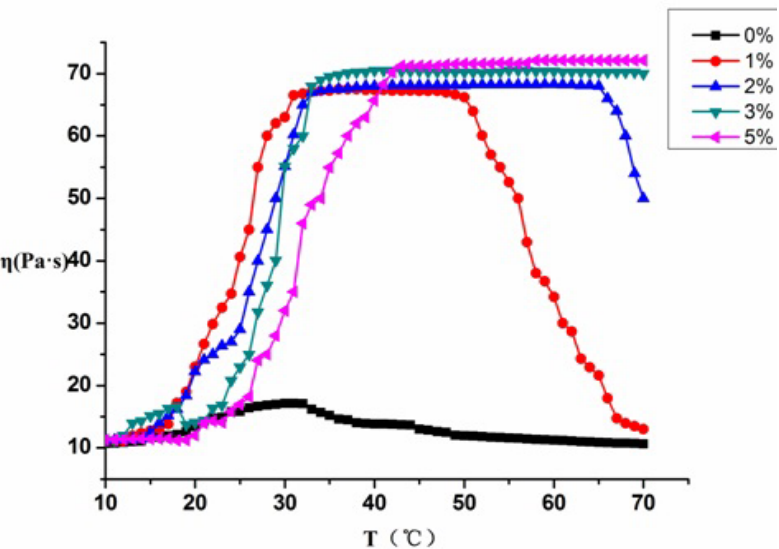

d

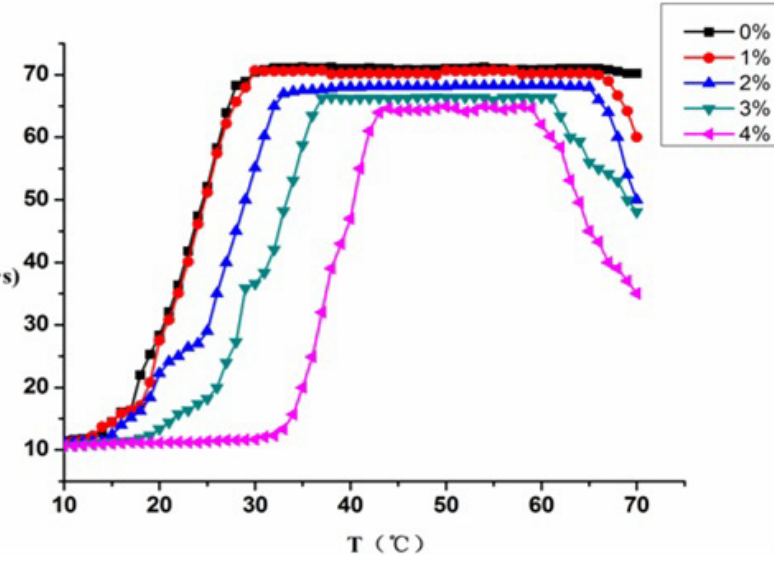

f

Figure 4 shows that when the concentrations of P188 (4\%) and PEG6000 (2\%) remained constant and the concentration of $\mathrm{P} 407$ was $16 \%$ or $18 \%$, the solution was still liquid despite an increase in temperature. The gelling temperature did not significantly change, which meant that it was not a gel. 
This may be related to the concentration of P407, which was too low to make the micelles tangle and stack with each other.

When the concentration of P407 was $20 \%, 22 \%$ or $24 \%$, the gelling temperature showed a dependence on the P407 concentration: $T_{1}$ decreased, $T_{2}$ increased and the gel process grew wider as the P407 concentration increased. When the concentration of P407 was 24\%, $\eta$ did not change significantly after the temperature increased to the gelling temperature, $T_{1}$. When the concentration of P407 was 22\%, the gel process was wider than that of 20\% P407 and the number of micelles and the chance of contact and entanglement increased, favoring the formation of the gel. Therefore, $22 \% \mathrm{P} 407$ was selected for further study.

\subsubsection{The Effect of $\mathrm{P} 188$ on $T_{1}, T_{2}$ and $\eta$}

A certain amount of paeonol inclusion, 22\% P407 and 2\% PEG6000 were put into the solution. The effects of $0 \%, 1 \%, 2 \%, 3 \%$ or $5 \%(w / w)$ P188 on $T_{1}, T_{2}$ and $\eta$ were investigated. The results are shown in Figure 4c,d.

Figure 4 shows that when the P407 PEG6000 concentrations were kept constant (22\% and 2\%, respectively) and the $\mathrm{P} 188$ concentration was $0 \%$, the sample could form a basic gel. The viscosity of this sample was low, and the gelling temperature had no significant transition point. The state tended to be a solution and was not stable. When the P188 concentrations were $2 \%, 6 \%, 10 \%$ or $14 \%, T_{1}$ and $T_{2}$ showed an upward trend as the concentration of P188 increased, the gel process became wider and the gel became much more stable. This may be related to the increasing ability of the P407 and P188 copolymer to form micelles that tangle and stack easily with each other. When the P188 concentration reached $6 \%, 10 \%$ or $14 \%, T_{2}$ was high, the gel had a higher melting transition point and $\eta$ was also higher. However, when the $\mathrm{P} 188$ concentration was $2 \%$, a good gel could be formed at $33.5^{\circ} \mathrm{C}$ (the temperature of the nasal cavity is $33{ }^{\circ} \mathrm{C}-34{ }^{\circ} \mathrm{C}$ ) and the gel could melt at approximately $55{ }^{\circ} \mathrm{C}$. This may be related to the fact that P188 might be able to change the ratio of PEO and PPO in P407. Therefore, $2 \%$ P188 was selected for further study.

\subsubsection{The Effect of PEG6000 on $T_{1}, T_{2}$ and $\eta$}

A certain amount of paeonol inclusion, 22\% P407 and 2\% P188 were put into the solution. $T_{1}, T_{2}$ and $\eta$ were measured when the polyethylene glycol (PEG) concentration was $0 \%, 1 \%, 2 \%, 3 \%$ or $4 \%(w / w)$. Figure $4 \mathrm{e}, \mathrm{f}$ show the results of this study.

When the concentrations of P407 and P188 stayed the same (22\% and 2\%, respectively) and the concentration of PEG6000 was $0 \%$, the sample solution formed a gel at $31.3{ }^{\circ} \mathrm{C}$, which was lower than the optimal gel formation temperature of $33.5{ }^{\circ} \mathrm{C}$. When the PEG6000 concentration was $1 \%, 2 \%, 3 \%$ or $4 \%, T_{1}$ increased, $T_{2}$ decreased, the gel process gradually became narrower and $\eta$ decreased as the concentration of PEG6000 increased. When the concentration of PEG6000 was $2 \%, T_{1}$ remained at the optimum temperature of $33.5 \pm 0.5{ }^{\circ} \mathrm{C}, \eta$ remained at approximately $70 \mathrm{~Pa} \cdot \mathrm{s}$ and the gel was relatively stable. These results might be because the PEG molecules formed micelles with P407 after PEG6000 was added into the solution in which P407 was obviously soluble. Meanwhile, PEG significantly reduced the viscosity of the gel. Therefore, the best choice for PEG6000 concentration was 2\%. 
In summary, the optimal makeup of the paeonol temperature-sensitive in situ gel was as follows: 2\% paeonol, 22\% P407, 2\% P188 and 2\% PEG6000. Under the optimal makeup, the state of the temperature-sensitive in situ gel was good and was stable in three markers of validation, as $T_{1}, T_{2}$ and $\eta$ were $33.5 \pm 0.29{ }^{\circ} \mathrm{C}, 55 \pm 0.56{ }^{\circ} \mathrm{C}, 70 \pm 0.67 \mathrm{~Pa} \cdot \mathrm{s}$, respectively.

\subsection{In Vitro Release Study}

The cumulative permeation of paeonol from the paeonol thermosensitive in situ gel was calculated according to the following Equation [14]:

$$
Q=2 C_{0}\left(D_{\mathrm{t}} / \pi\right)^{1 / 2}
$$

In the formula, $Q$ is the cumulative release of paeonol from the thermosensitive in situ gel per unit area, $C_{0}$ is the initial concentration, $D$ is the diffusion coefficient and $t$ is the time.

The cumulative in vitro paeonol release is shown in Figure 5, which shows that the cumulative in vitro paeonol release gradually increased as time increased. The drug release was fast during the first $12 \mathrm{~h}$ and relatively stable during the remaining $12 \mathrm{~h}$. The level of paeonol released within $12 \mathrm{~h}$ was in line with the Higuchi equation $\left(Q=147.5 t^{1 / 2}-58.01, r=0.9990\right)$ by curve fitting. The curve showed that the cumulative release of paeonol was proportional to $t^{1 / 2}$ within $12 \mathrm{~h}$ and was approximately proportional to $t$ during the remaining time. It could be said that the inclusion complex of paeonol and $\beta-C D$ could be absorbed into body through the mucous membranes and have some sustained effect.

Figure 5. The cumulative in vitro release of paeonol from the temperature-sensitive in situ gel into a saline receiving solution.

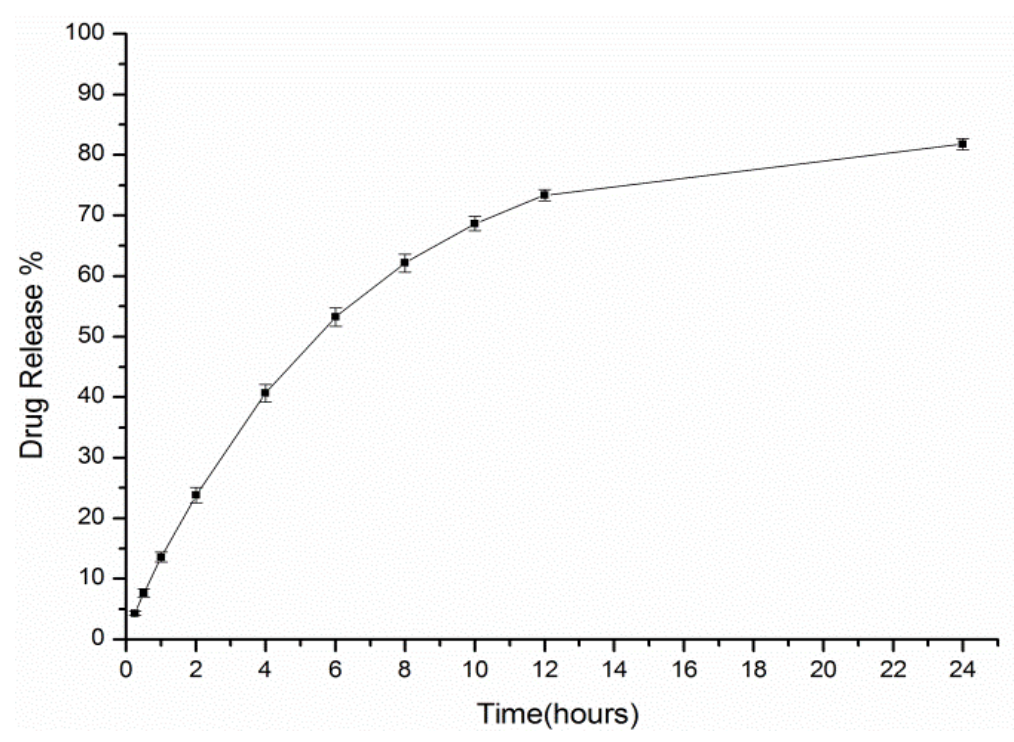

\subsection{Nasal Ciliary Toxicity Study of the Paeonol Temperature-Sensitive In Situ Gel}

Nasal cilia were observed under the microscope and the results shown in Figure 6. The cilia of the physiological saline group (Group A) and ephedrine hydrochloride group were clear, complete and lined up in order and moved actively and regularly swayed in the same direction (shown in 
Figure 6a,c). However, the cilia in the above groups stopped swinging, and most of their edge broke away from the nasal mucosa after treatment with sodium deoxycholate (shown in Figure 6b). It has been suggested that sodium deoxycholate has a serious destructive effect on mucosal tissues and a serious, irreversible inhibitory effect on ciliary movement. The cilia of the paeonol administration groups and the blank temperature sensitive gel group were clear, and their movement was active (shown in Figure 6d,e). This study demonstrated that the drugs and auxiliary materials had little toxicity on nasal cilia and that they could be used in a nasal delivery system. The length of continuous cilia swinging and the relative percentages of time are shown in Table 1.

Figure 6. Observations of the cilia on the nasal mucosa of the toad palate. The photographs were taken at a magnification of $400 \times$. (a) Physiological saline group; (b) $1 \%$ sodium deoxycholate group; (c) Naristillae Furacilini et Ephedrini group; (d) Paeonol temperature-sensitive in situ gel group; (e) Blank temperature-sensitive in situ gel group. Scale: $50 \mu \mathrm{m}$

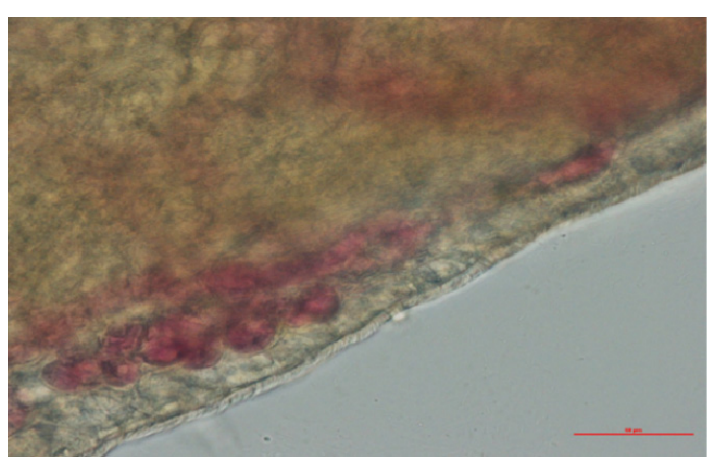

(a)

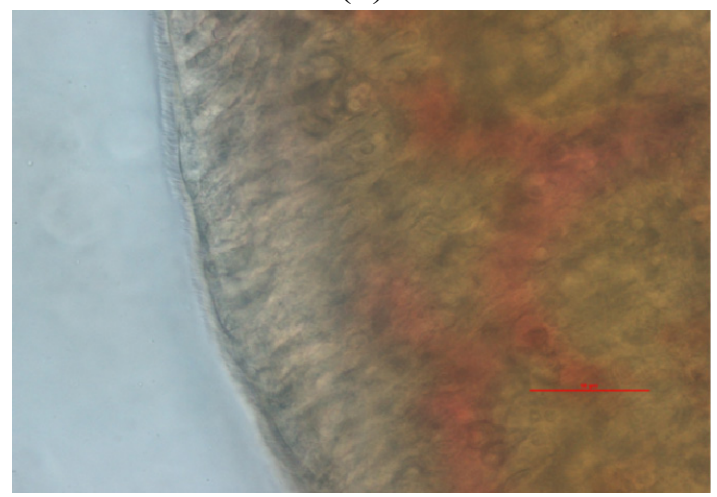

(c)

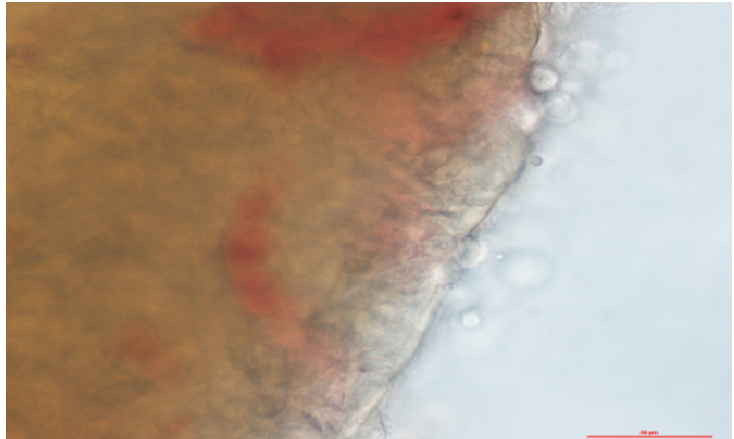

(b)

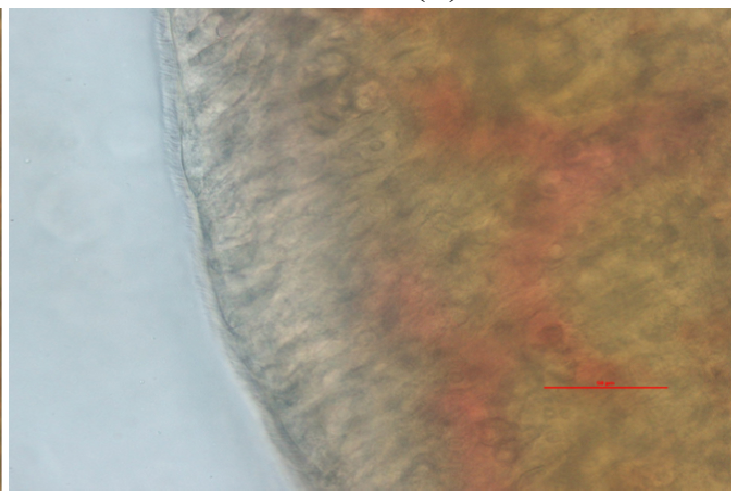

(d)

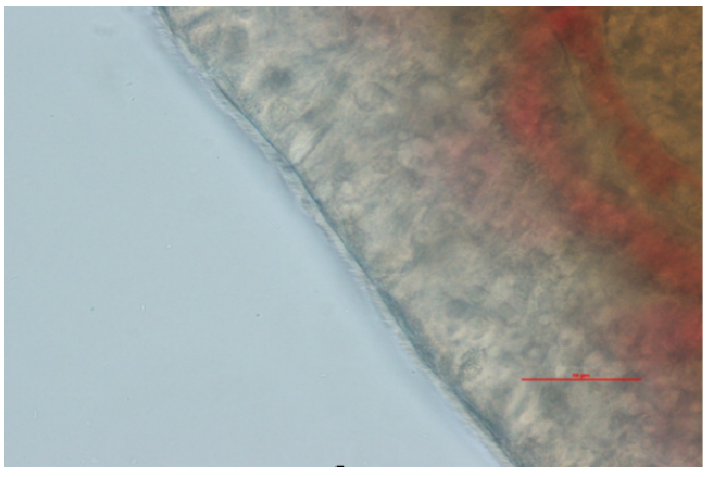

(e) 
Table 1. Time of cilia continuing swinging of paeonol temperature-sensitive in situ gel $(n=6)$.

\begin{tabular}{|c|c|c|c|c|c|}
\hline Items & $\begin{array}{c}\text { Physiological } \\
\text { saline }\end{array}$ & $\begin{array}{c}\text { Ephedrine } \\
\text { hydrochloride }\end{array}$ & $\begin{array}{c}\text { 1\% Sodium } \\
\text { deoxycholate }\end{array}$ & $\begin{array}{c}\text { Blank temperature- } \\
\text { sensitive in situ gel }\end{array}$ & $\begin{array}{l}\text { Paeonol temperature- } \\
\text { sensitive in situ gel }\end{array}$ \\
\hline $\begin{array}{c}\text { Continuing } \\
\text { swinging time } \\
\text { of cilia (min) }\end{array}$ & $701.60 \pm 32.7$ & $694.43 \pm 28.21$ & $33.4 \pm 5.7 *$ & $676.20 \pm 31.3$ & $662.60 \pm 30.1$ \\
\hline $\begin{array}{c}\text { Relative } \\
\text { percentage (\%) }\end{array}$ & $100.00 \%$ & $98.98 \%$ & $4.76 \% *$ & $96.38 \%$ & $94.44 \%$ \\
\hline
\end{tabular}

Note: relative percentage $=$ time of experimental group/time of negative control group $\times 100 ; *$ data for $p<0.01$

\subsection{Studies of the Paeonol Temperature-Sensitive In Situ Gel for the Treatment of Allergic Rhinitis}

\subsubsection{IgE and LTE4 in the Serum}

The results of the leukotriene E4 (LTE4) and immunoglobulin E (IgE) serum assays are shown in Table 2. The IgE and LTE4 levels in the sera of sensitized guinea pigs (group B to group F) were significantly higher than the IgE and LTE4 levels in the sera of non-sensitized guinea pigs (group A) $(p<0.05)$. Therefore, it could be deduced that our model of allergic rhinitis was accurate. Because the IgE and LTE4 levels in the sera of the paeonol temperature-sensitive in situ gel group were significantly lower than those of the model group, it was suggested that the paeonol temperature-sensitive in situ gel may suppress allergic reactions by reducing IgE and LTE4 levels.

Table 2. Content of leukotriene E4 (LTE4) and immunoglobulin E (IgE) in serum of allergic rhinitis (AR) guinea pigs in each group.

\begin{tabular}{|c|c|c|c|}
\hline Groups & Number of animals & LTE4 (pg/mL) & $\operatorname{IgE}(\mathrm{pg} / \mathrm{mL})$ \\
\hline Blank control group (group A) & 10 & $5.37 \pm 2.81$ & $0.71 \pm 0.45$ \\
\hline AR model group (group B) & 10 & $220.88 \pm 133.32$ & $141.38 \pm 85.06^{\# \#}$ \\
\hline Rhinocort nasal spray group (group C) & 10 & $32.65 \pm 5.60^{\#^{*}}$ & $18.98 \pm 6.93^{\# *}$ \\
\hline $\begin{array}{l}\text { Low-dose of paeonol temperature-sensitive } \\
\text { in situ gel group (group D) }\end{array}$ & 10 & $16.63 \pm 4.81^{\#^{*}}$ & $7.72 \pm 2.06^{\#^{*}}$ \\
\hline $\begin{array}{l}\text { Middle-dose of paeonol temperature-sensitive } \\
\text { in situ gel group (group E) }\end{array}$ & 10 & $11.70 \pm 2.43^{\# * *}$ & $3.46 \pm 1.78^{\# * *}$ \\
\hline $\begin{array}{l}\text { High-dose of paeonol temperature-sensitive } \\
\qquad \text { in situ gel group (group F) }\end{array}$ & 10 & $10.37 \pm 3.03^{\# * *}$ & $3.16 \pm 2.54^{\# * *}$ \\
\hline
\end{tabular}

Note - compare with blank control group: ${ }^{\#}$ data for $p<0.05$; ${ }^{\# \#}$ data for $p<0.01$. Compare with model group: * data for $p<0.05 ; * *$ data for $p<0.01$.

\subsubsection{Histological Examination of Nasal Mucosa}

An examination of the guinea pig nasal mucosal sections indicated that the nasal mucosal structures of the blank control group were intact and there was no oedema, vascular congestion or inflammatory cell infiltration in the submucosa (as shown in Figure 7a). The model group showed obvious oedema, a loose organizational structure, vascular congestion, inflammatory cell infiltration and large numbers of eosinophils (as shown in Figure 7b). The oedema, vascular congestion and eosinophil infiltration into 
the nasal mucosa were significantly reduced in the paeonol temperature-sensitive in situ gel groups and the positive control group (as shown in Figure $7 \mathrm{~d}-\mathrm{f}$ ).

Figure 7. Pathological changes in guinea pig nasal mucosal tissues. (a) blank control group; (b) AR model group; (c) Rhinocort nasal spray group; (d) low-dose paeonol- $\beta-C D$ temperature-sensitive in situ gel group; (e) middle-dose paeonol temperature-sensitive in situ gel group; (f) high-dose paeonol temperature-sensitive in situ gel group. Scale: $50 \mu \mathrm{m}$

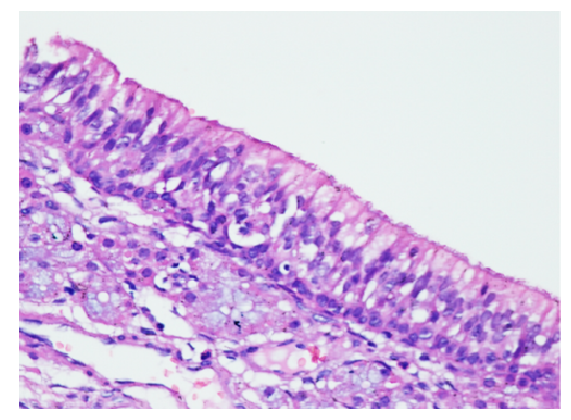

(a)

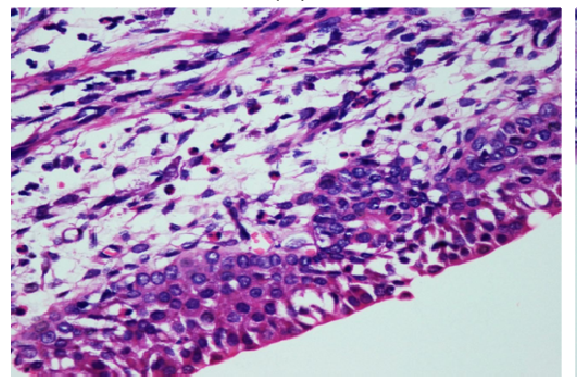

(d)

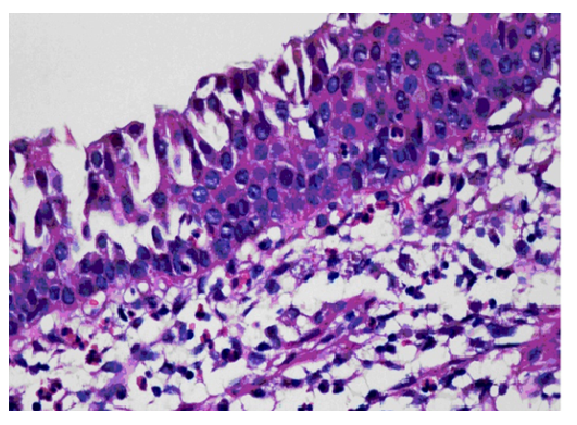

(b)

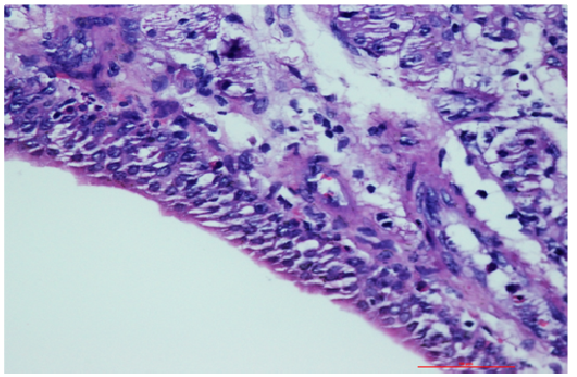

(e)

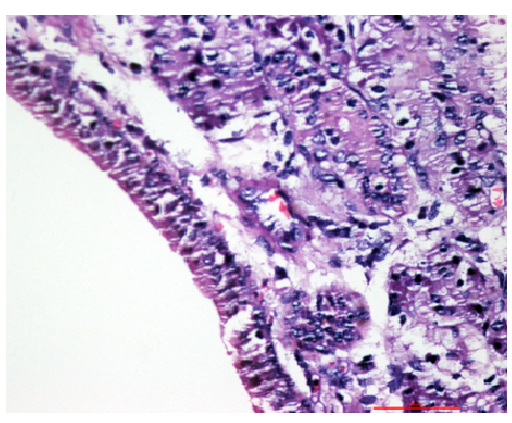

(c)

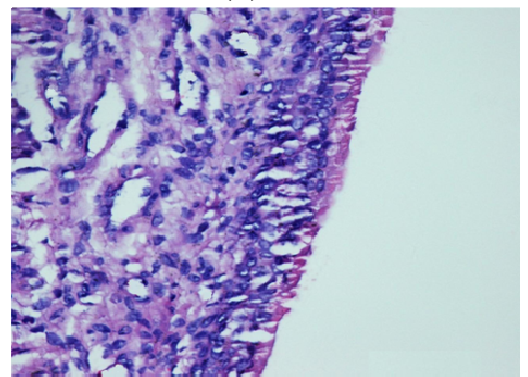

(f)

The eosinophil counts for the nasal mucosa of each group are shown in Table 3. The number of eosinophils increased significantly after allergen priming in Group B, Group C, Group D, Group E and Group F. The number of eosinophils in the medication groups was significantly reduced in comparison to the model group. These results are similar to the results of the IgE and LTE4 assays, suggesting that the paeonol temperature-sensitive in situ gel may treat RA by reducing inflammatory cell infiltration in the local nasal mucosa.

Table 3. Number of eosinophil in the nasal mucosa of AR guinea pigs in each group.

\begin{tabular}{ccc}
\hline Groups & Number of animals & Number of eosinophil in the nasal mucosa \\
\hline Blank control group (group A) & 10 & $4.18 \pm 1.66$ \\
AR model group (group B) & 10 & $6.46 \pm 2.16^{\# \#}$ \\
Rhinocort nasal spray group (group C) & 10 & $3.60 \pm 0.81^{\# \#^{* *}}$ \\
Low-dose of paeonol temperature-sensitive & 10 & $4.60 \pm 2.28^{\# \#^{*}}$ \\
$\quad$ in situ gel group (group D) & 10 & $3.36 \pm 1.83^{\# \#^{* *}}$ \\
Middle-dose of paeonol temperature-sensitive & & $3.89 \pm 1.32^{\# * *}$ \\
in situ gel group (group E) & 10 & \\
High-dose of paeonol temperature-sensitive & &
\end{tabular}

Note - compare with blank control group: ${ }^{\#}$ data for $p<0.05$; ${ }^{\#}$ data for $p<0.01$. Compare with model group: * data for $p<0.05 ; * *$ data for $p<0.01$. 


\section{Experimental Section}

\subsection{Materials}

Poloxamer 407 and poloxamer 188 were purchased from BASF Corporation, Germany. The PEG6000 and $\beta$-CD were purchased from Sinopharm Group Co., Ltd. (Shanghai, China). The sodium deoxycholate was purchased from Solarbio Technology Co., Ltd. (Beijing, China). The ovalbumin (egg, grade V) was purchased from Sigma-Aldrich Co. LLC. (Saint Louis, MO, USA). Methanol was purchased from Merck Company (Frankfurter Str., Darmstadt, Germany). Double-distilled deionized water was used in this paper.

A dialysis bag (MWCO 6000) was bought from USA Viskase Corporation, the CDR-34P differential scanning calorimeter was from Shanghai Precision Scientific Instrument Co., Ltd. (Shanghai, China). The FTIR 8400-S type of Fourier transform infrared spectrometer was purchased from Shimadzu, Japan. The NDJ-8S-type rotating viscometer was purchased from Shanghai Precision Scientific Instrument Co., Ltd. The XS105 electronic analytical balance was from METTLER TOLEDO. The RYJ-6A transdermal drug diffusion test instrument was bought from Shanghai Yellow Sea Drug Testing Instrument Co., Ltd. (Shanghai, China). The Nikon DS-Ril biological digital microscope was from Nikon Instrument Co., Ltd. (Tokyo, Japan).

\subsection{Preparation of an Inclusion Complex of Paeonol and $\beta-C D$}

In this study, a saturated aqueous solution method was employed to prepare the inclusion complex of paeonol and $\beta-C D[15,16]$. An adequate amount of paeonol was accurately weighed and sewn up together into $\beta$-cyclodextrin at a proportion of $1: 8$ at $45^{\circ} \mathrm{C}$ for $1 \mathrm{~h}$ and was then refrigerated for $24 \mathrm{~h}$ at $4{ }^{\circ} \mathrm{C}$ to crystallize. The filtrate and the inclusion were obtained from this preparation.

\subsection{The Characterization of the Paeonol- $\beta$-CD Complex}

\subsubsection{Infrared Spectroscopic Analysis}

Infrared spectroscopic analysis of the paeonol- $\beta$-CD complex was done, as per the literature [17]. Potassium bromide was used for tabletting. The medical grade paeonol, $\beta-\mathrm{CD}$, the physico-compounds of paeonol and $\beta-C D$ and the inclusion complex of paeonol and $\beta-C D$ were analyzed by infrared spectrometry.

\subsubsection{Differential Scanning Calorimetry (DSC) Analysis}

Differential scanning calorimetry (DSC) analysis of the paeonol- $\beta$-CD complex was done, as per the literature [18]. Five milligrams of paeonol, $\beta-C D$, the physico-compounds of paeonol and $\beta-C D$ and the inclusion complex of paeonol and $\beta-\mathrm{CD}$ were weighed precisely and placed into the crucible of the DSC analyzer. Then, they were determined at $10{ }^{\circ} \mathrm{C} / \mathrm{min}$ of the heating rate in air. $\alpha-\mathrm{A}_{2} \mathrm{O}_{3}$ was used as a reference. The differential thermal curves for each of the samples were compared. 


\subsection{Preparation of the Paeonol Temperature-Sensitive In Situ Gel}

An appropriate cold process method [19] was employed to prepare the inclusion complex of paeonol and $\beta-C D$ in the in situ gel. Prescribed dose of paeonol inclusion, P188 and PEG6000 were added to deionized water, stirred, dissolved and then mixed with $\mathrm{P} 407$. The solution was stored overnight in a refrigerator at $4{ }^{\circ} \mathrm{C}$ until the $\mathrm{P} 407$ was completely dissolved and the paeonol in situ gel was obtained.

\subsection{Evaluation of the Paeonol Temperature-Sensitive In Situ Gel}

\subsubsection{Thermodynamic Study (Gelling Temperature $T_{1}$ and Gel Melting Temperature $T_{2}$ )}

In this study, $T_{1}$ and $T_{2}$ were determined by a magnetic stirrer with 85-2 type (Changzhou, China) [20-22]. Ten grams of temperature-sensitive in situ gel solution and a stir bar were added to a vial. A micro thermometer with an accuracy of $0.1{ }^{\circ} \mathrm{C}$ was also inserted into the vial with the mercury bulb immersed in the gel solution. Its correction coefficient was 1.006 . The vial was then put into a water bath set at $10^{\circ} \mathrm{C}$, and the rotation speed was set at $300 \mathrm{r} / \mathrm{min}$. The temperature raising speed was maintained between $1{ }^{\circ} \mathrm{C} / \mathrm{min}$ and $2{ }^{\circ} \mathrm{C} / \mathrm{min}$. Afterwards, the temperature at which the magnetic stir bar completely stopped rotating was defined as the gelling temperature $\left(T_{1}\right)$. The temperature at which the magnetic stir bar could start rotating with continuing heating was defined as the gel melting temperature $\left(T_{2}\right)$. The preparation was a semisolid gel from $T_{1}$ to $T_{2}$. This temperature range was defined as the gel process. The thermodynamic variation of the paeonol thermosensitive gel was evaluated by the determination of $T_{1}$ and $T_{2}$. All of the samples were measured three times in parallel, with the results expressed as the mean of the three readings.

\subsubsection{Rheological Properties Study}

The viscosity of the paeonol thermosensitive in situ gel was measured by a rotational viscometer (NDJ-8S, Shanghai, China) [23]. One hundred milliliters of thermosensitive in situ gel that was stored at $4{ }^{\circ} \mathrm{C}$ was put into a beaker and the gel was heated to $40{ }^{\circ} \mathrm{C}$ by a controllable water bath (HH-S, Shanghai, China). The viscosity was measured every $1{ }^{\circ} \mathrm{C}$. All of the studies were performed three times in parallel.

\subsection{In Vitro Release Study}

The Franz diffusion cell method [24] (RYJ-6A, Shanghai, China) was used to determine the release of the paeonol thermosensitive in situ gel in vitro. The semi-permeable membrane (molecular weight cut-off 6000, USA Viskase Corporation, Illinois, USA) was placed between the diffusion cell and the receiving cell. Then, $0.5 \mathrm{~g}$ of gel was placed into the diffusion cell and evenly distributed on the semi-permeable membrane surface with an area of $0.75 \mathrm{~cm}^{2}$. The membrane was then placed in an oven at $34{ }^{\circ} \mathrm{C}$ for $10 \mathrm{~min}$ and a gel was achieved. The diffusion cell was then placed in a $34 \pm 0.1^{\circ} \mathrm{C}$ water bath, the receiving cell was filled with warm saline solution and a speed of $300 \mathrm{rpm}$ was maintained with a stir bar. All of the receiving fluid was removed, and the same amount of new receiving fluid was quickly added at $0.25,0.5,1,2,4,6,8,10,12$ and $24 \mathrm{~h}$. The receiving fluid was filtered through a $0.45 \mu \mathrm{m}$ microporous membrane, and the first part of the filtrate was discarded. 
High-performance liquid chromatography (HPLC) [23] was used to determinate the peak area of paeonol and to calculate the paeonol content. These experiments were performed three times in parallel.

\subsection{Nasal Ciliary Toxicity Study}

The in situ toad palate model was used to study the nasal ciliary toxicity of the paeonol temperature-sensitive in situ gel $[25,26]$. In this experiment, $0.5 \mathrm{~mL}$ paeonol thermosensitive in situ gel was dripped onto the palate mucosa after exposing the toad palate. The drug was washed off by physiological saline after contacting the palate for $30 \mathrm{~min}$. The palate mucosa were then separated, washed and placed under an optical microscope to watch the movement of the cilia. Ephedrine hydrochloride, which is generally acknowledged to be safe for cilia, was set as the negative control. Sodium deoxycholate, which is generally acknowledged as having severe ciliotoxicity, was chosen as the positive control drug. Physiological saline was chosen as the blank control.

\subsection{Preliminary Pharmacodynamic Study}

\subsubsection{Animals}

Forty-eight guinea pigs (specific pathogen free (SPF), Hartley guinea, half male and half female, 300-400 g) were maintained under specific pathogen free (SPF) conditions. The temperature was controlled to $25{ }^{\circ} \mathrm{C} \pm 0.2{ }^{\circ} \mathrm{C}$, and the relative humidity was controlled at $55 \% \pm 5 \%$. We performed these experiments in the Animal Centre of the Fujian University of Traditional Chinese Medicine.

\subsubsection{Grouping and Modeling}

All of the guinea pigs were acclimated for 5 days before being divided randomly into group A, B, C, D, E or F $(n=8)$. Group A was used as the physiological saline group (blank control group), group B was used as the model group, group $\mathrm{C}$ was used as the Rhinocort nasal spray group, group D was used as the low-dose paeonol temperature-sensitive in situ gel group, group E was used as the middle-dose paeonol temperature-sensitive in situ gel group and group F was used as the high-dose paeonol temperature-sensitive in situ gel group. Every guinea pig, except those in the blank control group, was intraperitoneally injected with modeling suspension (which was made up of $0.5 \mathrm{mg}$ of ovalbumin (OVA) as the antigen, $30 \mathrm{mg}$ of aluminum hydroxide powder as the adjuvant and $1 \mathrm{~mL}$ of physiological saline) once a day for 7 days. Fifty microliters of $2 \%$ egg albumen was then placed in their nasal cavities once a day for five days to elicit a reaction. The guinea pigs in the blank control group were given the equivalent volume of physiological saline in the same way.

\subsubsection{Administration and Sample Collection}

Administration began on the second day of the seven sensitizing injections. Fifty microliters of physic liquor was dripped into the guinea pigs' nasal cavities three times daily and was given for 11 continuous days. The guinea pigs in the blank control group were given an equivalent volume of physiological saline in the same way. After eleven days, all of the guinea pigs were fasted overnight. The guinea pigs were then anaesthetized with ketamine-diazepam by intraperitoneal injection, and the 
blood was aseptically obtained from the abdominal aorta. The blood-containing tubes were allowed to stand at room temperature for $2 \mathrm{~h}$, and the sera were obtained by centrifugation at $3000 \times g$ for $20 \mathrm{~min}$ at $4{ }^{\circ} \mathrm{C}$ and stored at $-80{ }^{\circ} \mathrm{C}$. After blood collection, the turbinate and septal mucosa were obtained and fixed in $10 \%$ formalin.

\subsubsection{Determination of IgE and LTE4 in Serum}

The serum levels of IgE and LTE4 were measured using enzyme-linked immunosorbent assay (ELISA) kits (Xitang, Shanghai, China), according to the manufacturer's instructions. The wells were coated with $100 \mu \mathrm{L}$ capture antibody diluted in coating buffer. The plate was sealed and incubated overnight at $4{ }^{\circ} \mathrm{C}$. After three washes, the wells were blocked with $200 \mu \mathrm{L}$ assay diluent at room temperature for $1 \mathrm{~h}$, followed by other washes. Then, $100 \mu \mathrm{L}$ diluted IgE and LTE4 standard and test samples were added and incubated for $2 \mathrm{~h}$ at room temperature. After repeated washes, the substrate was added and incubated for $20 \mathrm{~min}$ at room temperature, and the absorbance was measured at $450 \mathrm{~nm}$ using an ELISA reader (BioTek, Model ELX800, Chicagoland, IL, USA).

\subsubsection{Histopathological Examination of the Nasal Mucosa}

The nasal mucosa was taken out from the $10 \%$ formalin. Then was paraffin-embedded, sectioned and stained with hematoxylin and eosin (H\&E). Histopathological changes were observed under a light microscope, five visual fields were observed and eosinophil counts were recorded.

\subsection{Statistical Analysis}

The data are shown as the mean \pm standard deviation (SD). The statistical analyses were performed using SPSS 16.0.

\section{Conclusions}

In this paper, the composition of the paeonol temperature-sensitive in situ gel was screened by thermodynamic and rheology studies, and the optimal composition was determined to be $2 \%$ paeonol inclusion, 22\% P407, 2\% P188 and 2\% PEG6000. Nasal toxicity and in vitro release were also investigated, and the results of these studies showed that the paeonol thermosensitive gel had low ciliary toxicity and could be used for intranasal administration. The in vitro release data were in line with the Higuchi equation, indicating that the paeonol could be absorbed into body through the mucous membranes and have some sustained effect. Finally, the preliminary efficacy of the paeonol thermosensitive in situ gel was evaluated with the guinea pig RA model. These results suggested that the paeonol thermosensitive in situ gel can significantly reduce $\operatorname{IgE}$ and $\mathrm{LTE}_{4}$ levels and the number of eosinophils, in addition to improving the pathological changes in the nasal mucosa of the guinea pig. This study provides the basis and a new direction for the treatment of allergic rhinitis. 


\section{Acknowledgments}

This work was supported by the Nature Science Foundation of China (NO.81073117), the National High Technology Research and Development Program of China (863 Program) (NO.2007AA021810) and the Science and Technology Innovation Platform Projects of the Fujian Province of China (2010Y2004).

\section{References}

1. Schenkel, E.J. Effect of desloratadine on the control of morning symptoms in patients with seasonal and perennial allergic rhinitis. Allergy Asthma Proc. 2006, 27, 465-472.

2. Lu, B. New Techniques and New Dosage Forms of Drugs, 1st ed.; People's Medical Publishing House: Beijing, China, 2002; p. 451.

3. Gizurason, S. The relevance of nasal physiology to the design of drug absorption studies. Adv. Drug Deliv. Rev. 1993, 11, 329-347.

4. Zhang, C.X.; Zhang, W.T.; Wang, D.K. The research progress of in situ gel, new type of drug delivery system. Chin. J. Hosp. Pharm. 2006, 26, 459-461.

5. Ning, L.L. Characteristics of ophthalmic in situ gel and issues in progress. Chin. J. New Drugs 2007, 16, 988-990.

6. Schmolka, I.R. Poloxamers in the pharmaceutical industry. In Polymers for Controlled Drug Delivery; Tarcha, P.J., Ed.; CRC Press: Boca Raton, FL, USA, 1991; pp. 189-214.

7. Chou, T.C. Anti-inflammatory and analgesic effects of paeonol in carrageenan evoked thermal hyperalgesia. Br. J. Pharmacol. 2003, 139, 1146-1152.

8. Ma, Y.L.; Bates, S.S.; Gurney, A.M. The effects of paeonol on the electrophysiological properties of cardiac ventricular myocytes. Eur. J. Pharmacol. 2006, 545, 87-92.

9. Kim, J.; Lee, H.; Lee, Y.; Oh, B.G.; Cho, C.; Kim, Y.; Shin, M.; Hong, M.; Jung, SK.; Bae, H. Inhibition effects of Moutan Cortex Radicis on secretion of eotaxin in A549 human epithelial cells and eosinophil migration. J. Ethnopharmacol. 2007, 114, 186-193.

10. Kim, S.H.; Kim, S.A.; Park, M.K., Kim, S.H.; Park, Y.D.; Na, H.J.; Kim, H.M.; Shin, M.K.; Ahn, K.S. Paeonol inhibits anaphylactic reaction by regulating histamine and TNF-a. Int. Immunopharmacol. 2004, 4, 279-287.

11. Tang, H.Y.; Yang, S.; Wang, J.B. Verview of preparation process, formulation reform and clinical application of paeonol. Jiangsu J. Trad. Chin. Med. 2004, 25, 58-60.

12. Hirlekar, R.; Kadam, V. Preformulation study of the inclusion complex irbesartan- $\beta$-cyelodextrin. AAPS PharmSciTech 2009, 10, 276-281.

13. Song, T.; Wang, D.K.; Gao, H.; Zhang, C.X.; Ji, B. Thermodynamic and rheological properties of therm osensitive gels for nasal delivery of ribavirin. Chin. J. Pharm. 2006, 37, 540-542.

14. De Santana, D.C.; Pupo, T.T.; Sauaia, M.G.; da Silva, R.S.; Lopez, R.F.V. Nitric oxide photorelease from hydrogels and from skin containing a nitro-ruthenium complex. Int. J. Pharm. 2010, 391, 21-28.

15. Jug, M.; BEeIREvIe-Lacan, M.; Bengez, S. Novel cyclodextrin-based film form ulation intended for buecal delivery of atenolol. Drug Dev. Ind. Pharm. 2009, 35, 796-807.

16. $\mathrm{Xu}, \mathrm{X} . \mathrm{Y}$. The Identification of principal components of clathrate and complex with infrared spectral subtraction method. Chin. J. Pharm. Anal. 1997, 17, 87-89. 
17. Lu, W.Y.; Chen, P.R.; Lin, G.Q. New stereoselective synthesis of thiamphenicol and florfenicol from enantiomerically pure cyanohydrin: A chemo-enzymatic approach. Tetrahedron 2008, 64, 7822-7827.

18. Schmolka, I.R. Artificial skin I. Preparation and properties and properties of pluronic F-127 gels for the treatment of burns. J. Biomed. Mater. Res. 1972, 6, 571-582.

19. Mao, S.; Shi, Z.; Bi, D. Research of nasal absorption of dipyrone solution. Chin. Pharm. J. 1997, 32, 87-90.

20. Song, C.J.; Wang, Y.; Wang, C.Y. Preparation and evaluation in vivo of scutellaria thermosensitive gel. China J. Chin. Mat. Med. 2008, 33, 628-631.

21. Miyazaki, S.; Nakamura, T.; Takada, M. Thermo-sensitive sol-gel transition of Pluronic F-127. Yakuzaigaku 1991, 51, 36-43.

22. Azade, T.; Fatemeh, A.; Rassoul, D. Temperature-responsive and biodegradable PVA: PVP k30: poloxamer 407 hydrogel for controlled delivery of human growth hormone (hGH). J. Pediatr. Endocr. Met. 2011, 24, 175-179.

23. Gratieri, T.; Gelfuso, G.M.; Rocha, E.M.; Sarmento, V.H.; de Freitas, O.; Lopez, R.F.V. A poloxamer/chitosan in situ forming gel with prolonged retention time for ocular delivery. Eur. J. Pharm. Biopharm. 2010, 75, 186-193.

24. National Pharmacopoeia Committee. Chinese Pharmacopoeia, 1st ed.; TCM Science \& Technology Publishing House: Beijing, China, 2010; pp. 268-269.

25. Jiang, X.G.; Cui, J.B.; Fang, X.L.; Wei, Y.; Xi, N.Z. Toxicity of drugs on nasal mucocilia and the method of its evaluation. Acta Pharm. Sin. 1995, 308, 848-853.

26 Zhang, Q.; Jiang, X.; Xiang, W.; Lu, W.; Su, L.; Shi, Z. Preparation of nimodipineloaded microemulsion for intranasal delivery and evaluation of the targeting efficiency to brain. Int. J. Pharm. 2004, 275, 85-96.

(C) 2013 by the authors; licensee MDPI, Basel, Switzerland. This article is an open access article distributed under the terms and conditions of the Creative Commons Attribution license (http://creativecommons.org/licenses/by/3.0/). 